\title{
Ionic conductivity and hopping rate data for some NASICON analogues
}

\author{
N B DESAI*, K BYRAPPA, A B KULKARNI* and \\ G S GOPALAKRISHNA \\ Department of Geology, University of Mysore, Manasagangotri, Mysore 570 006, India \\ * Department of Physics, Gulbarga University, Gulbarga 585 106, India
}

MS received 30 December 1986; revised 20 March 1987

\begin{abstract}
The a.c. conductivity of ionic materials shows two regions of frequency-dependent conductivity over a wide range of frequencies. Jonscher's law of dielectric response for ionic conductors enables us to characterize the conductivities. The region of low frequency dispersion approximates to a frequency-independent plateau enabling us to obtain the d.c. conductivity. In some other conductors, the presence of low-frequency dispersion cannot be neglected while determining the effective d.c. conductivity. We have used this method to extract the d.c. conductivity and hopping rate as well as to estimate concentrations of the mobile ions (carriers) in some NASICON analogues.
\end{abstract}

Keywords. Nasicon analogues; superionic conductors; solid electrolytes; hopping rate; frequency dispersion; jump frequency; mobile ion concentration.

\section{Introduction}

Superionic materials are being extensively investigated in recent years in view of their potential value as solid electrolytes in solid state battery systems. The ionic conductivity and other related characteristics are usually studied by a.c. techniques to avoid the necessity of developing non-blocking electrodes needed for d.c. measurements. Recently it has been suggested that a.c. conductivity data could be used to extract the hopping rates of ions in superionic materials. This technique has been applied on $\mathrm{Na} \beta^{\prime \prime}$-alumina, $\mathrm{LiGaO}_{2}$ and $\mathrm{Ag} / \mathrm{Na}$-mixed alkali $\beta$-alumina single crystals. These investigations enable us to study the role of carrier mobility and mobile ion concentrations in determining the net ionic conductivity of a particular material (Almond et al 1982, 1983, 1984; Almond and West 1985). In the present paper similar studies have been carried out on NASICON analogues.

NASICON (sodium superionic conductor $-\mathrm{Na}_{1+{ }_{x}} \mathrm{Zr}_{2} \mathrm{Si}_{x} \mathrm{P}_{3-x} \mathrm{O}_{12}, \mathrm{O}<x<3$ ) has been the most popular material for many years. Owing to problems connected with the synthesis, lack of stoichiometry in the final products, lack of single crystals, zirconium deficiency etc NASICON analogues (preferably pure phosphates having more simple structures and stoichiometric in composition), in the form of single crystals are being preferred.

The dielectric loss $\chi^{\prime \prime}(\omega)$ and the real part of the dielectric constant $\chi^{\prime}(\omega)$ can be expressed as follows (Jonscher 1978):

$$
\begin{aligned}
& \chi^{\prime \prime}(\omega) \propto\left(\omega / \omega_{p}\right)^{n_{1}-1}+\left(\omega / \omega_{p}\right)^{n_{2}-1}, \\
& \chi^{\prime \prime}(\omega) \equiv \sigma(\omega) / \varepsilon_{0}(\omega),
\end{aligned}
$$

for the hopping ion conductors and conducting solids (dipolar dielectrics) where $\omega_{p}$ denotes the characteristic frequency of the dielectric loss peak. The change in the 
conductive response from low to high frequency dispersion occurs where $\omega \approx \omega_{p}$. Using (1) and (2), and by identifying $\omega_{p}$ with the thermally activated (with an activation energy $E_{a}$ ) ion hopping rate, one can express the d.c. conductivity (Huggins 1975) as

$$
\sigma(0)=K \omega_{p}=\left[N e^{2} a^{2} / k T\right] \gamma C(\mathrm{i}-c) \omega_{p} .
$$

The magnitude of the constant $K=\sigma(0) / \omega_{p}$ is largely a measure of the mobile ion concentration. $\gamma$ is a geometrical factor that may include a correlation factor, $C$ is the concentration of mobile ions on $N$ equivalent lattice sites per unit volume, $a$ is the hopping distance and $e$ is the electronic charge. The thermally-activated mobile ion concentration $C$ is the basis of the Arrhenius relation for the superionic materials. One can further analyze the a.c. conductivity as a function of the measuring frequency $\omega$ as expressed by the following equation:

$$
\sigma(\omega)=K \omega_{p}^{1-n_{1}} \omega^{n_{1}}+K \omega_{p}^{1-n_{2}} \omega^{n_{2}}
$$

The plots of $\log _{10} \sigma(\omega)$ vs $\log _{10} \omega$ give slopes $n_{1}$ and $n_{2}$ which are non-zero in most materials studied by us. This contradicts the earlier assumption $n_{1}=0$ and $n_{2}=n$ (Hill and Jonscher 1979). The tangents of $\log _{10} \sigma(\omega)$ vs $\log _{10} \omega$ plots intersect at $\omega=\omega_{p}$, where the conductivity equals $2 \sigma(0)$. Hence one can extract the d.c. conductivity from the a.c. conductivity data. Further analysis of d.c. conductivity $\sigma(0)$ enables an assessment of the carrier mobility and mobile ion concentration in the superionic material. Based on the values of $n_{1}$ and $n_{2}$, one can classify the materials into three categories (i) those with no conductivity plateau, (ii) those with welldeveloped conductivity plateau and (iii) those with intermediate conductivity plateau.

\section{Synthesis}

NASICON analogues- $\mathrm{NaNi}_{2} \mathrm{ZrP}_{3} \mathrm{O}_{12}, \quad \mathrm{Na}_{2}(\mathrm{La}, \mathrm{Co}) \mathrm{TiP}_{3} \mathrm{O}_{12}$ and $\mathrm{Na}_{2}(\mathrm{La}, \mathrm{Al})$ $\mathrm{TiP}_{3} \mathrm{O}_{12}$ were synthesized in the form of small single crystals according to the method described earlier (Byrappa and Gopalakrishna 1985; Byrappa et al 1985, 1986).

\section{Complex impedance measurements}

The complex impedance measurements were carried out according to the technique reported earlier (Hodge et al 1976). The NASICON analogue crystals were powdered and pressed into pellets by applying $77.2 \mathrm{MN} / \mathrm{m}^{2}$ pressure. The pellets $(10 \mathrm{~mm}$ dia, $3 \mathrm{~mm}$ thick and 85 to $90 \%$ compactness) were provided with blocking silver electrodes on either side. These pellets were sintered in a vacuum chamber $\left(10^{-2}\right.$ torr) at $300^{\circ} \mathrm{C}$. A capacitance bridge (General Radio 1620-B) was used in the present work. The pellets were placed inside an evacuated $\left(10^{-2}\right.$ torr) tubular furnace (with a three-terminal arrangement) whose temperature could be controlled with an accuracy of $\pm 1{ }^{\circ} \mathrm{C}$. The ionic conductivity measurements were carried out within the frequency range d.c. to $1 \mathrm{MHz}$ and within the temperature range of room temperature to $330^{\circ} \mathrm{C}$. Low frequency dispersion, high frequency dispersion and electrode polarization are evident in the conductivity data. The corresponding log $\sigma(\omega)$ vs $\log \omega$ plots are given in figures 1 and 2 . The calculated values of $\omega_{p}, \sigma(o)$, $\sigma\left(\omega_{p}\right), n_{1}, n_{2}$ and $K$ are given in table 1 . 


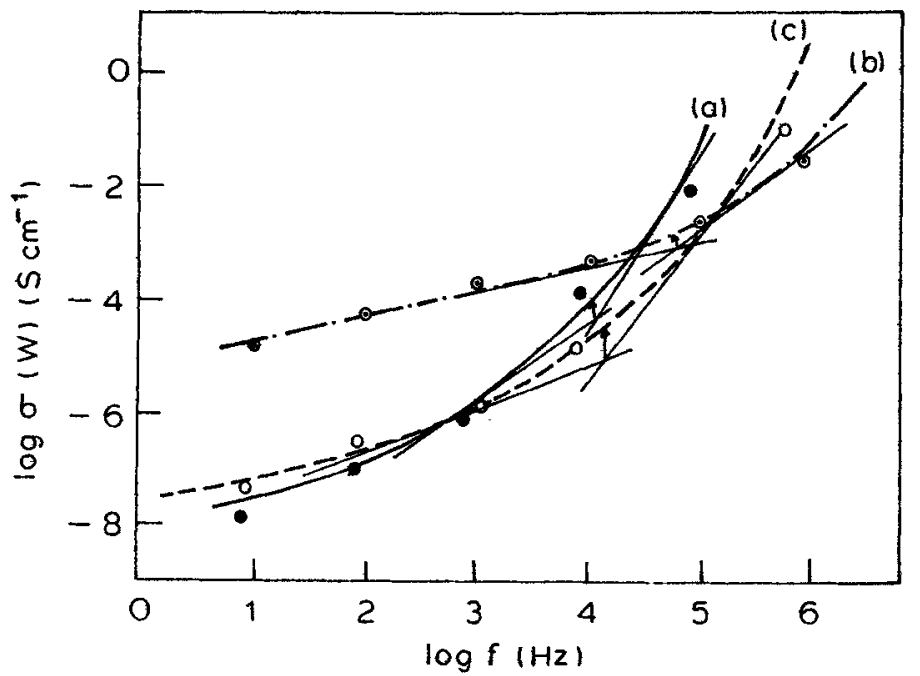

Figure 1. $\log \sigma(\omega)$ vs $\log \omega$ plot for (a) $\mathrm{NaNi}_{2} \mathrm{ZrP}_{3} \mathrm{O}_{12}$ at $60^{\circ} \mathrm{C}$. (b) $\mathrm{NaNi}_{2} \mathrm{ZrP}_{3} \mathrm{O}_{12}$ at $300^{\circ} \mathrm{C}$ and (c) $\mathrm{Na}_{2}(\mathrm{La}, \mathrm{Al}) \mathrm{TiP}_{3} \mathrm{O}_{12}$ at $85^{\circ} \mathrm{C}$.

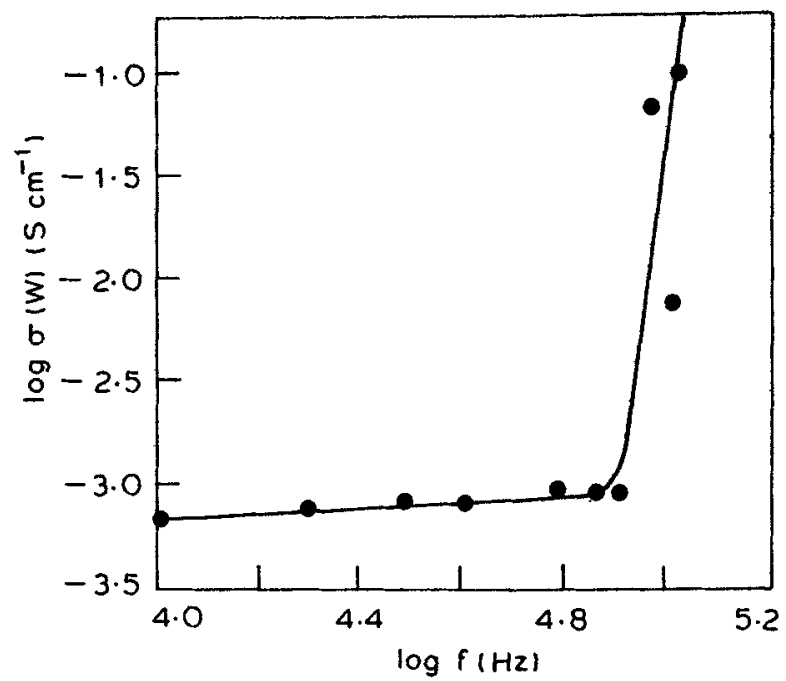

Figure 2. $\log \sigma(\omega)$ vs $\log \omega$ plot for $\mathrm{Na}_{2}(\mathrm{La}, \mathrm{Co}) \mathrm{TiP}_{3} \mathrm{O}_{12}$ at $220^{\circ} \mathrm{C}$.

Table 1. Hopping tate data for NASICON analogues

\begin{tabular}{lrccccccc}
\hline Compound & \multicolumn{1}{c}{$\begin{array}{c}\omega_{p} \\
\left({ }^{\circ} \mathrm{C}\right)\end{array}$} & \multicolumn{1}{c}{$\begin{array}{c}\omega_{p} \\
(1 / 2)\end{array}$} & $\begin{array}{c}\sigma(0) \\
\left(\mathrm{S} \mathrm{cm}^{-1}\right)\end{array}$ & $\begin{array}{c}\left(\omega_{p}\right) \\
\left(\mathrm{S} \mathrm{cm}^{-1}\right)\end{array}$ & \multicolumn{1}{c}{$n_{1}$} & $n_{2}$ & $\begin{array}{c}K \\
(\Omega \mathrm{mHz})^{-1}\end{array}$ & $\begin{array}{c}E_{a} \\
(\mathrm{eV})\end{array}$ \\
\hline $\mathrm{NaNi}_{2} \mathrm{ZrP}_{3} \mathrm{O}_{12}$ & 60 & 634333 & $3.1 \times 10^{-5}$ & $6.3 \times 10^{-5}$ & 0.8 & 1.0 & $4.8 \times 10^{-10}$ & 0.6 \\
& 300 & 100000 & $1.99 \times 10^{-4}$ & $5.01 \times 10^{-4}$ & 0.4 & 1.06 & $1.9 \times 10^{-9}$ & 0.6 \\
$\mathrm{Na}_{2}(\mathrm{La}, \mathrm{Co}) \mathrm{TiP}_{3} \mathrm{O}_{12}$ & 220 & 75857 & $7.58 \times 10^{-4}$ & - & 0 & 4.0 & $9.98 \times 10^{-9}$ & $0 \cdot 7$ \\
$\mathrm{Na}_{2}(\mathrm{La}, \mathrm{Al}) \mathrm{TiP}_{3} \mathrm{O}_{12}$ & 85 & 10000 & $1.0 \times 10^{-5}$ & $2.51 \times 10^{-5}$ & 0.6 & 1.13 & $1.0 \times 10^{-10}$ & 0.62 \\
\hline
\end{tabular}




\section{Discussion}

Almond et al (1984) reported two distinct regions of low and high frequency dispersion at high and low temperature regions for $\mathrm{Na} \beta^{\prime \prime}$-alumina. In the case of $\mathrm{LiGaO}_{2}$ and $\mathrm{Na} / \mathrm{Ag} \beta$-alumina, the corresponding regions were displayed at $856 \mathrm{~K}$ (and below) and $294 \mathrm{~K}$ (and below) respectively. Such low and high frequency dispersions have been observed in most of our materials at various temperatures (figures 1 and 2).

The slopes of the plots of $\log \sigma(\omega)$ vs $\log \omega$ in the high frequency dispersion regions vary from 1.00 to 1.50 . The values of $n_{2}$ correspond to $n_{2}=2$ for an electrode response that may be simulated by a Debye-like series $\mathrm{RC}$ element. The change in the conductivity response from low to high frequency dispersion occurs at $\omega \approx \omega_{p}$. This region shifts to higher frequencies with increasing temperature, thus confirming the thermal activation of $\omega_{p}$.

The magnitude of the constant $K\left(K=\sigma(0) / \omega_{p}\right.$ equation (2)) is a measure of the mobile ion concentration. From table 1, it is seen that the values of $K$ range from $10^{-12}$ to $10^{-9} \Omega^{-1} \mathrm{~cm}^{-1} \mathrm{~Hz}^{-1}$. Almond et al (1984) reported the following values of $K$ for different materials:

$$
\begin{array}{ll}
\mathrm{Na} \beta^{\prime \prime} \text {-alumina } & 1.5 \times 10^{-12} \Omega^{-1} \mathrm{~cm}^{-1} \mathrm{~Hz}^{-1}, \\
\mathrm{LiGaO}_{2} & 1.5 \times 10^{-14} \Omega^{-1} \mathrm{~cm}^{-1} \mathrm{~Hz}^{-1}, \\
\mathrm{Na} / \mathrm{Ag} \beta \text {-alumina } & 5.0 \times 10^{-13} \Omega^{-1} \mathrm{~cm}^{-1} \mathrm{~Hz}^{-1}
\end{array}
$$

The NASICON analogues reported in this work show relatively higher mobile ion concentrations.

Based on the values of $n_{1}$ and $n_{2}$, the NASICON analogues have been classified into categories I and II mentioned by Almond et al (1984). We have observed that $\mathrm{NaNi}_{2} \mathrm{ZrP}_{3} \mathrm{O}_{12}\left(\right.$ at $90^{\circ} \mathrm{C}$ ) and $\mathrm{Na}_{2}(\mathrm{La}, \mathrm{Co}) \mathrm{TiP}_{3} \mathrm{O}_{12}$ belong to category I; surprisingly, $\mathrm{NaNi}_{2} \mathrm{ZrP}_{3} \mathrm{O}_{12}$ becomes a material pertaining to category II at $300^{\circ} \mathrm{C}$. Such a phenomenon could be explained in terms of variation in jump distance $a$, geometrical correlation factor and the number of available equivalent sites $N$ per unit volume for a particular superionic as a function of temperature. Larger $K$ values in the NASICON analogues of this work indicate to some extent a higher jump frequency, larger jump distance and a higher correlation factor. In the high frequency dispersion region, the power-law dispersion is attributed to the presence of manybody excitations in the superionic system (Ngai et al 1979; Dissodo and Hill 1979). The low frequency dispersion involves translational motion of ions. This dispersion may be caused by the presence of impurities and crystalline imperfections that impede the motion of ions through the lattice (Jonscher 1978). Yoshikado et al (1982) have shown that the low frequency dispersion in hollandite is dependent on crystalline perfection, whereas Dissado and Hill (1983) attribute the low frequency dispersion to the restricted ionic motion, i.e. in one or two dimensions. On the other hand, the high frequency dispersion and high ionic conductivity in NASICON analogues vis-a-vis $\mathrm{Na} \beta$-alumina can be attributed to a cooperative event involving many neighbouring ions. Dygas and Brodwin (1986) carried out measurements on the frequency dependent conductivity of NASICON ceramics in the microwave region showing a lower charge correlation factor $f_{c}=\sigma(0) / \sigma(\omega)$ for low temperature regions.

The hopping rates for NASICON analogues vary in the order of $10^{4}$ to $10^{5}$ which 
is slightly lower than that of $\mathrm{Na} \beta^{\prime \prime}$-alumina single crystals. This may be due to the polycrystalline nature of the NASICON analogues. The grain boundaries in polycrystalline material may decrease the hopping rate.

The values of d.c. conductivity in NASICON analogues appear in general to be smaller than that of $\mathrm{Na} \beta^{\prime \prime}$-alumina. By further appropriate substitutions (for example, the replacement of $\mathrm{Ti}$ with $\mathrm{Zr}$ and $\mathrm{Hf}$ ) of various elements within the framework of NASICON analogues, one can achieve higher conductivity., This work is in progress and will be reported separately.

The low frequency dispersion in the dielectric loss $\chi^{\prime \prime}(\omega)$ and the real part of the dielectric constant, $\chi^{\prime}(\omega)$ are related by the Kramers-Kronig relation:

$$
\chi^{\prime \prime}(\omega) / \chi^{\prime}(\omega)=\operatorname{Cot}\left(n_{1} \pi / 2\right) \text {. }
$$

Appreciable values of $n_{1}$ for some of the NASICON analogues (table 1) indicate smaller $\chi^{\prime \prime} / \chi^{\prime}$ values. Larger $\chi^{\prime \prime}$ values indicate higher absorption of energy by the dielectric material. Small values of $n_{1}$ in some of NASICON analogues indicate that the material does not absorb energy and instead allows it to flow through, which is an essential and important feature of a superionic conductor.

\section{References}

Almond D P and West A R $1985 \mathrm{~J}$. Electroanal. Chem. 18617

Almond D P, Duncan G K and West A R 1983 Solid State Ionics 8159

Almond D P, Hunter C C and West A R 1984 J. Mater, Sci. 193236

Almond D P, West A R and Grant R J 1982 Solid State Commun. 441277

Byrappa K and Gopalakrishna G S 1985 Prog. Cryst. Growth Charact. 1189

Byrappa K, Gopalakrishna G S. Kulkarni A B and Venkatachalapathy V 1985 J. Less Com. Metals 110 441

Byrappa K, Kulkarni A B and Gopalakrishna G S 1986 J. Mater, Sci. Lett. 5408,519

Dissado L A and Hill R M 1979 Nature (London) 279685

Dissado L A and Hill R M 1983 Private Communication

Dygas J R and Brodwin M E 1986 Solid State Ionics 18/19 981

Hill R M and Jonscher A K 1979 J. Non-Cryst. Solids 3253

Hodge J M, Ingran M D and West A R 1976 J. Electroanal. Chem. 74125

Huggins R A 1975 Diffusion in solids, recent developments (eds) A S Nowick and $J$ Academic Press)

Jonscher A K 1978 Philos. Mag. B38 587

Ngai K L, Jonscher A K and White C T 1979 Nature (London) 277185

Yoshikado S, Ohachi T, Taniguchi I, Onada Y, Watanabe M and Fuziki Y 1982 Solid State Ionics 7335 